\title{
Reactive hyperemia during early reperfusion as a determinant of improved functional recovery in ischemic preconditioned rat hearts
}

Annie Rochetaing, PhD

Paul Kreher, PhD

From the Laboratoire de Préconditionnement et de Remodelage du Myocarde, UFR Sciences, Angers Cedex, France.

Received for publication July 26, 2002; revisions requested Sept 27, 2002; revisions received Oct 8, 2002; accepted for publication Oct 22, 2002

Address for reprints: Annie Rochetaing, Laboratoire de Préconditionnement et de Remodelage du Myocarde, UFR Sciences, 2 Boulevard Lavoisier, F-49045, Angers Cedex, France. (E-mail: annie.rochetaing@ univ-angers.fr).

J Thorac Cardiovasc Surg 2003;125: 1516-25

Copyright (C) 2003 by The American Association for Thoracic Surgery

$0022-5223 / 2003 \$ 30.00+0$

doi:10.1016/S0022-5223(03)00024-2
Objective: Our study was undertaken to clarify the impact of the shear stressinduced reactive hyperemia (associated with reperfusion) in preconditioning-mediated protection.

Methods: In control rat hearts, a 40-minute preischemic perfusion (constant pressure: $70 \mathrm{~mm} \mathrm{Hg}$ ) period was followed by 25 -minute global low-flow ischemia (constant flow: $0.3 \mathrm{~mL} / \mathrm{min}$ ) and 30 -minute reperfusion (constant pressure). As preconditioning protocol, hearts underwent 2 cycles of 5-minute no-flow ischemia/ 5-minute reperfusion.

Results: Although coronary vasodilation in response to shear stress is severely impaired after global low-flow ischemia and reperfusion, it is fully preserved by ischemic preconditioning concomitantly with an improvement of left ventricular developed pressure. Restricting coronary peak flow to $100 \%$ of baseline at reperfusion reduced left ventricular recovery to the control level. $\mathrm{N}^{\mathrm{G}}$-nitro-L-arginine methyl ester affects the restoration of reperfusion-reactive hyperemia and the improvement of contractile recovery afforded by ischemic preconditioning. However, if the time course of hyperemia was restored by forcibly reperfusing to $150 \%$ of baseline for 10 minutes and, therefore, by restricting final peak flow to $80 \%$ of baseline for 20 minutes, contractile function recovered to a high degree despite the presence of $\mathrm{N}^{\mathrm{G}}$-nitro-L-arginine methyl ester.

Conclusion: We conclude that wall stretch and shear stress during reperfusion are necessary for the mediation phase of preconditioning.

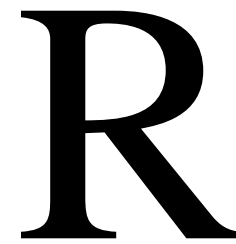

eactive hyperemia is a well-known phenomenon in which coronary artery occlusion and subsequent reperfusion elevate the coronary blood flow above the baseline level determined prior to occlusion. ${ }^{1}$ This vascular reactivity has been investigated extensively and may involve a variety of mechanisms and mediators, including myogenic relaxation, ${ }^{2}$ adenosine, ${ }^{3}$ prostanoids, ${ }^{4}$ nitric oxide (NO) ${ }^{5}$ endothelin, ${ }^{6}$ and adenosine triphosphate (ATP)-sensitive $\mathrm{K}\left(\mathrm{K}_{\mathrm{ATP}}\right)$ channels. ${ }^{7}$ Although evaluated at the beginning of reperfusion, this elevation was considered to be a fundamental indication of the preservation of vessel muscle function. Kuo and Chancellor ${ }^{8}$ reported that vascular smooth muscle cells in coronary arterioles can respond directly to wall stretch caused by change in intraluminal pressure (myogenic responses) and that endothelial cells can sense change in blood flow (flowmediated responses). However, the consequences of reperfusion depend on the intensity and duration of the preceding ischemia. ${ }^{9}$

Several experimental studies have demonstrated that repeated brief coronary artery ischemia-reperfusion cycles protect the heart from irreversible injury during subsequent longer episodes of ischemia. ${ }^{10}$ While most of the experiments showing this "preconditioning" modeled occlusion-reperfusion using a vascular clamp, 
Ovize and colleagues ${ }^{11}$ demonstrated this phenomenon using a coronary cycle flow variation model, which is closer to human spontaneous unstable angina. Moreover, clinical evidence $^{12}$ suggests that ischemic preconditioning may be induced by balloon inflation during percutaneous transluminal coronary angioplasty: the results show that the third inflation itself produced larger reactive hyperemia while producing less ischemic responses compared with the first inflation. Furthermore, it has been effectively reported that $\mathrm{NO}$ and/or adenosine play an important role in ischemic preconditioning ${ }^{13}$ : this suggests that both ischemic preconditioning and reactive hyperemia may be mediated by common mechanisms. Two subsequent phases are now recognized in the mechanism of ischemic preconditioning: a triggering phase and a mediation phase. ${ }^{13}$ Attention has been performed on the length of the transient ischemia, the number of brief ischemia-reperfusion cycles required to elicit an optimal preconditioning. Some works have been done on the influence of the delay between the last transient and sustained ischemia ${ }^{14,15}$ but few studies have focused on the correlation between increased reactive hyperemia during the first minutes of reperfusion and functional recovery of preconditioned hearts. Others have considered that the preconditioning effect seems to be linked to the repayment of the flow debt in the perfused rat heart ${ }^{16}$ and seems to develop fully only when reflow is complete in the in situ dog heart. ${ }^{11}$ We feel that this suggestion is both an important and relevant new consideration in the "memory" of preconditioning.

Therefore, this study was designed to determine the detailed time course of coronary flow (CF) throughout reperfusion following global low-flow ischemia in control and preconditioned hearts. In order to test that $\mathrm{NO} /$ cyclic guanosine monophosphate system may be an important modulator in the mediation phase of preconditioning, NO synthesis was blocked by $\mathrm{N}^{\mathrm{G}}$-nitro-L-arginine methyl ester (L-NAME) in some experiments to try to attenuate hyperemia during reperfusion. If $\mathrm{NO}$ plays a role in preconditioning and hyperemia, whether this means that hyperemia is necessary for preconditioning is the key question. To answer this, groups of hearts with low spontaneous hyperemia have been forcibly reperfused to give a high hyperemia, and groups of hearts with high spontaneous hyperemia have been reperfused at constant restricted flow.

\section{Experimental Procedure}

Female Wistar rats (Depre Elevage, BP 70, 18230 St Doulchard) weighing 240-300 g, maintained on standard diet (A04 pellets; UAR, Villemoisson/Orge), were used. The procedures in this study were in accordance with the guidelines and recommendations given by the French "Ministère de l'Agriculture et de la Forêt, France," on the use and care of animals. All animals were randomly divided into 8 groups.

\section{Isolated Rat Heart Apparatus/Langendorff Perfusion}

The isolated Langendorff-perfused rat heart preparation was used. The rats were anesthetized with sodium pentobarbital $(100 \mathrm{mg} / \mathrm{kg}$ intraperitoneal) and then injected with heparin $(1500 \mathrm{UI} / \mathrm{kg})$. Hearts were quickly excised and immersed in ice-cold Krebs-Henseleit buffer containing (in $\mathrm{mM}: \mathrm{NaCl}$ 118, $\mathrm{KCl}$ 5.6, $\mathrm{CaCl}_{2}$ 2.4, $\mathrm{MgCl}_{2}$ 1.2, $\mathrm{NaHCO}_{3} 20, \mathrm{Na}_{2} \mathrm{HPO}_{4}$ 1.2 , and glucose 11) to stop the contraction at once. Within 1 minute, aortic perfusion $(70 \mathrm{~mm} \mathrm{Hg}$ ) was initiated using the above solution gassed with $95 \% \mathrm{O}_{2} / 5 \% \mathrm{CO}_{2}(\mathrm{pH} 7.4)$ at $37^{\circ} \mathrm{C}$. The perfusate did not recirculate. The myocardial temperature was monitored constantly by a thermistor probe.

\section{Experimental Protocols}

The experimental protocols used are shown in Figure 1 (part $\mathrm{A}$ and part B).

All hearts were perfused for 20 minutes to establish equilibrium hemodynamics.

(1) In the ischemic control group (CTR, $n=6$ ), perfusion was prolonged for 20 minutes. Therefore, hearts were subjected, via a peristaltic pump (Minipulse 3 pump-Gilson), to a 25-minute global low-flow ischemia (at a constant flow of $0.3 \mathrm{~mL} / \mathrm{min}$ ), referred to as sustained ischemia. During reperfusion, perfusion pressure again was set at $70 \mathrm{~mm} \mathrm{Hg}$ for 30 minutes.

(2) Ischemic preconditioned hearts $(\mathrm{PC}, \mathrm{n}=6)$ were subjected to two rounds of 5-minute global no-flow ischemia $\left(\mathrm{GI}_{1}\right.$ and $\left.\mathrm{GI}_{2}\right)$, each followed by 5 -minute reperfusion $\left(\mathrm{R}_{1}\right.$ and $\left.\mathrm{R}_{2}\right)$,. This preconditioning protocol was followed by 25-minute low-flow ischemia and 30-minute reperfusion with constant pressure equal to $70 \mathrm{~mm} \mathrm{Hg}$.

(3) In the PC/100 group, the preconditioning protocol was the same as in the PC group, but reperfusion following low-flow ischemia was performed at a constant flow of $10.8 \pm 0.7 \mathrm{~mL} \cdot \mathrm{min}^{-1} \cdot \mathrm{g}^{-1}$ (equal to $100 \%$ of the baseline $\mathrm{CF}$ ) via a peristaltic pump for 30 minutes.

(4) In the PC/150 group, the preconditioning protocol was the same as in the protocols (2) and (3), but reperfusion following low-flow ischemia was performed at a constant flow of $16.5 \pm 0.5 \mathrm{~mL} \cdot \mathrm{min}^{-1}$. $\mathrm{g}^{-1}$ (equal to $150 \%$ of the baseline $\mathrm{CF}$ ) for 30 minutes.

(5), (6), (7) These three protocols were identical to protocols (2), (3) and (4) respectively, with in addition, continuous administration of L-NAME $\left(10^{-5} \mathrm{M}\right)$ during the 25-minute low-flow ischemia and 30minute reperfusion periods.

(8) In the PC/150 + 80/L-NAME group, the protocol was the same as in group (7) but, during reperfusion, constant flow of $150 \%$ was performed only for 10 


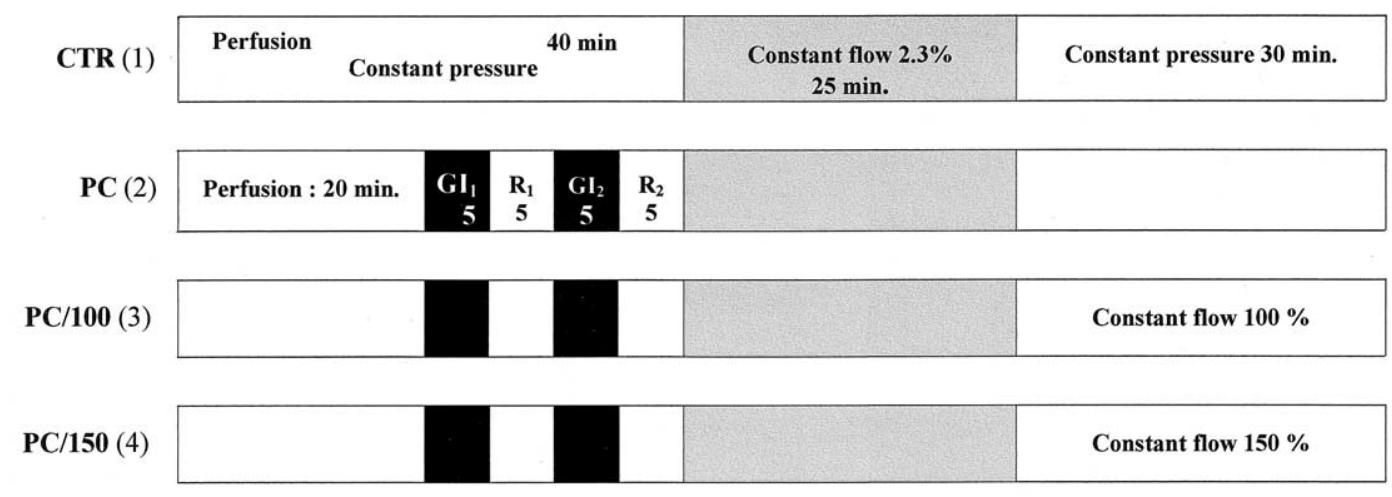

A
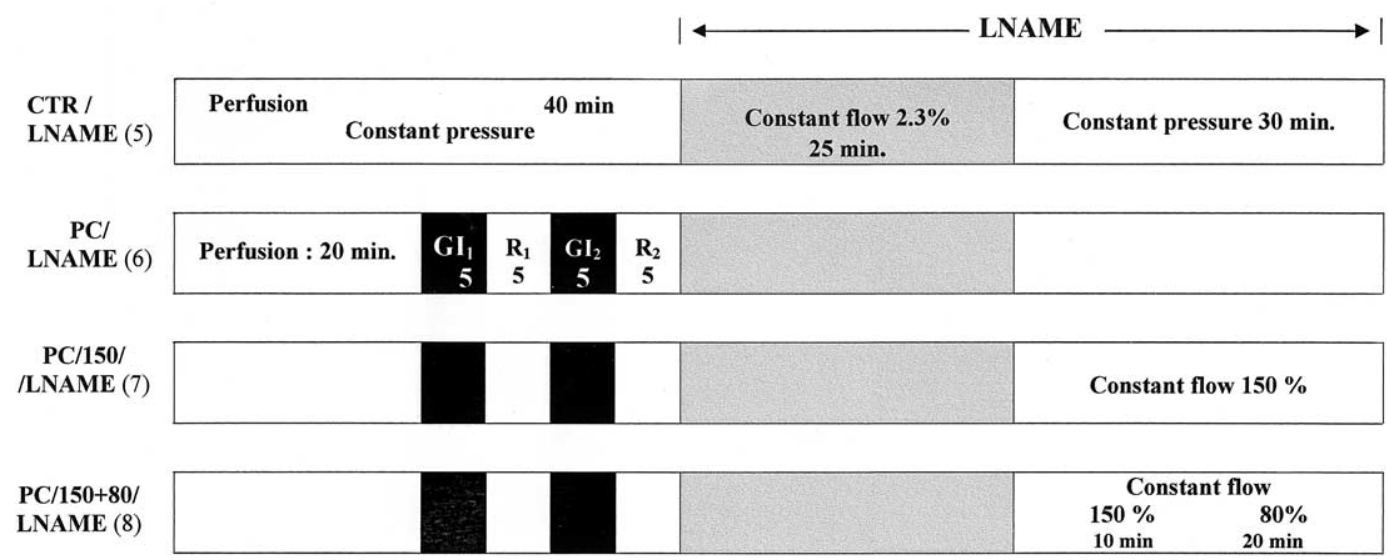

B

Figure 1. Schematic diagram of the experimental protocols used. All protocols (part A and part B) included an initial 20-minute stabilization period (at constant pressure $70 \mathrm{~mm} \mathrm{Hg}$ ), a 25-minute global low-flow ischemia (at constant flow $0.3 \mathrm{~mL} / \mathrm{min}$ ) followed by a 30 -minute reperfusion. In the first group (CTR), initial stabilization perfusion was prolonged to $\mathbf{4 0}$ minutes before low-flow ischemia, reperfusion was at constant pressure. After the 20-min stabilization period all the preconditioned groups (PC, PC/100, PC/150, PC/L-NAME, PC/150/L-NAME, and PC/150+80/ L-NAME) of hearts were subjected to 2 periods of 5 -minute global no-flow ischemia $\left(\mathrm{GI}_{1}, \mathrm{GI}_{2}\right)$, each followed by 5-minute reperfusion $\left(R_{1}, R_{2}\right)$. In the groups 5 to $8(B)$, L-NAME $\left(10^{-5} \mathrm{M}\right)$ was added to Krebs-Henseleit buffer during low-flow ischemia and final reperfusion periods. In group (3), peak flow was restricted to $100 \%$ of baseline with a peristaltic pump throughout reperfusion. In groups (4) and (7), hearts are forcibly reperfused to give a peak/baseline flow of $150 \%$ throughout reperfusion. In group (8), hearts are forcibly reperfused to $150 \%$ for 10 minutes and restricted to $\mathbf{8 0} \%$ for $\mathbf{2 0}$ minutes.

TABLE 1. Baseline parameters in the different groups of animals

\begin{tabular}{|c|c|c|c|c|c|}
\hline Groups & $\mathbf{n}$ & BW $(g)$ & LVDevP (mm Hg) & LVEDP $(\mathrm{mm} \mathrm{Hg})$ & $\mathrm{BCF}_{20}\left(\mathrm{~mL} \cdot \min ^{-1} \cdot \mathrm{g}^{-1}\right)$ \\
\hline CTR & 6 & $265 \pm 13$ & $137 \pm 19$ & $5 \pm 0.5$ & $11.1 \pm 0.8$ \\
\hline $\mathrm{PC}$ & 6 & $262 \pm 9$ & $129 \pm 13$ & $4.9 \pm 0.4$ & $11.4 \pm 0.8$ \\
\hline $\mathrm{PC} / 100$ & 6 & $265 \pm 12$ & $143 \pm 15$ & $5 \pm 0.5$ & $11.1 \pm 0.5$ \\
\hline $\mathrm{PC} / 150$ & 6 & $251 \pm 9$ & $133 \pm 11$ & $5 \pm 0.5$ & $10.9 \pm 0.4$ \\
\hline CTR/LNAME & 6 & $257 \pm 14$ & $139 \pm 17$ & $5.1 \pm 0.5$ & $11.1 \pm 1.0$ \\
\hline PC/LNAME & 6 & $263 \pm 10$ & $141 \pm 20$ & $4.9 \pm 0.5$ & $10.7 \pm 1.1$ \\
\hline PC/150/LNAME & 6 & $253 \pm 10$ & $131 \pm 12$ & $5 \pm 0.4$ & $10.9 \pm 0.4$ \\
\hline PC/150 + 80/LNAME & 6 & $249 \pm 12.5$ & $125 \pm 16$ & $5 \pm 0.4$ & $11.4 \pm 0.7$ \\
\hline
\end{tabular}

$\overline{B W}$, body weight; $L V D e v P$, left ventricular developed pressure; $L V E D P$, left ventricular end-diastolic pressure; $B C F_{20}$, baseline coronary flow at 20 minutes of the stabilization perfusion. 
minutes, followed by a constant flow equal to $80 \%$ of the baseline $\mathrm{CF}$ for 20 minutes.

\section{Functional Measurements}

In experiments, a compliant balloon was inserted into the left ventricle and inflated with water until a ventricular diastolic pressure of 4-5 mm Hg was recorded. This procedure resulted in systolic pressures of $100-160 \mathrm{~mm} \mathrm{Hg}$. Intraventricular pressure and heart rate were recorded via a Statham P23 XL pressure transducer.

Peak ischemic contracture was measured as the maximum rise in left ventricular end diastolic pressure from baseline values. Peak contracture was expressed as a percentage of preischemic left ventricular developed pressure ([LVDevP ] after 20-minute control perfusion).

$\mathrm{CF}$ was measured continuously by an electromagnetic blood flow and velocity meter MDL 1401 (Skalar Medical, Delft, The Netherlands) and stored on a paper polygraph. $\mathrm{BCF}$ was the baseline $\mathrm{CF}$ stabilized at the 20th minute. PCF was the peak coronary flow at the beginning of reperfusion and reactive hyperemia $(\mathrm{RH}=\mathrm{PCF} / \mathrm{BCF})$.

An epicardial electrocardiogram was recorded on a Gould polygraph throughout the experimental period. Ventricular tachycardia (VT) was defined as a run of four or more consecutive similar ventricular complexes and analyzed according to the Lambeth Conventions. ${ }^{17}$

\section{Electrical Activity}

Electrical recordings were obtained with floating glass microelectrodes filled with $3 \mathrm{M} \mathrm{KCl}$. Transmembrane potential was measured as the voltage difference between the intracellular electrode and an $\mathrm{Ag} / \mathrm{AgCl}$ ground electrode immersed in the fluid perfusing the heart. As myocardial action potential duration (APD) is influenced by cell location, we studied action potentials (AP) originating from the middle region of the left or right ventricle. Electrical activity was displayed on a digital oscilloscope. The duration of the $\mathrm{AP}$ was recorded at $90 \%$ repolarization $\left(\mathrm{APD}_{90}\right)$ using a Datapac system in a personal computer. Furthermore, VT was confirmed by the short RR interval and typical action potential amplitude.

\section{Drugs}

These experiments were performed with L-NAME from Sigma-Aldrich, Saint Quentin Fallavier, France.

\section{Data Analysis}

Functional and electrical activities were stored on an IBM 486 computer for subsequent analysis.

All the values were expressed as mean \pm SEM. Comparisons among the different groups were first made by analysis of variance, and post hoc analysis was done using

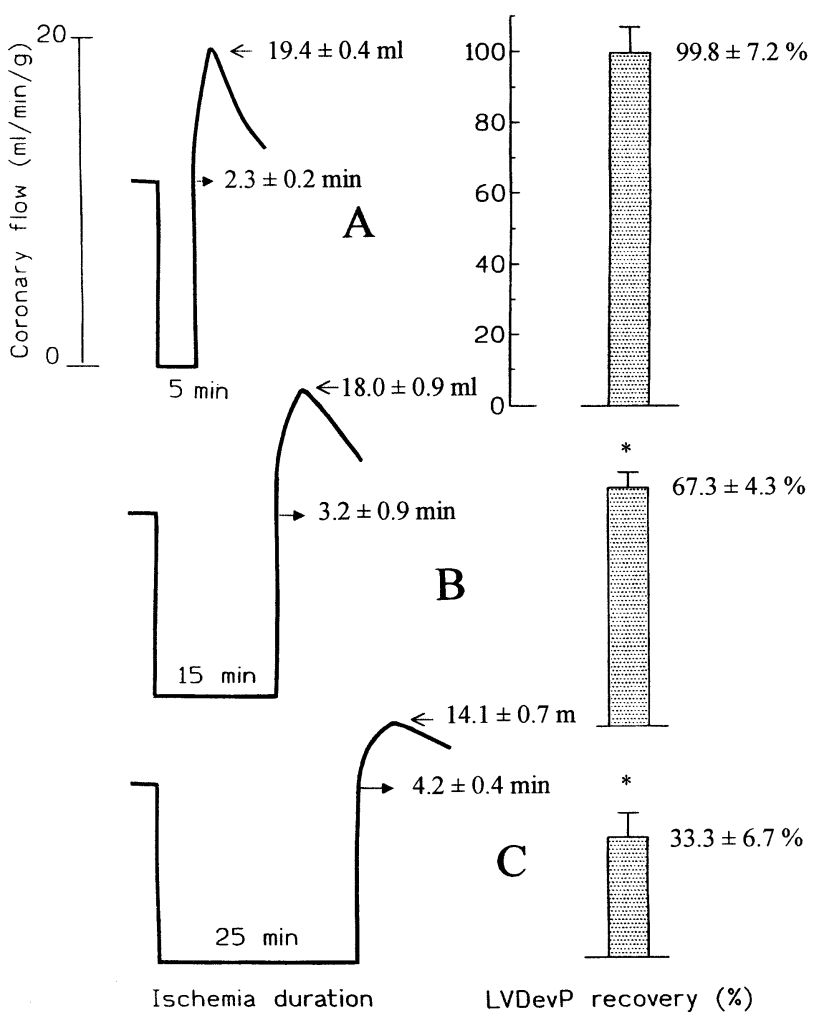

Figure 2. Coronary reactive hyperemia at the beginning of reperfusion after stabilization period and variable durations of global low-flow ischemia: A, 5 minutes; B, 15 minutes; C, 25 minutes. Histograms were the LVDevP recoveries, respectively, at the end of reperfusion. *Significant difference at $P<.05$ vs (A) group.

the Student-Newman-Keuls test. To compare VT incidence between the different groups, a $\chi^{2}$ test was used.

\section{Results}

\section{Baseline Hemodynamic Parameters}

No significant differences in left ventricular end-diastolic pressure, peak developed pressure, and CF were observed within or between groups during equilibrium periods (Table 1).

\section{Reactive Hyperemia and Duration of Ischemia}

The peak CF at the beginning of reperfusion was significantly delayed (from $2.3 \pm 0.2$ to $4.2 \pm 0.4 \mathrm{~min}$.) and reduced (from $19.4 \pm 0.4$ to $14.1 \pm 0.7 \mathrm{~mL} \cdot \mathrm{min}^{-1} \cdot \mathrm{g}^{-1}$ ) when the duration of ischemia increased from 5 to 25 minutes (Figure 2). Concomitantly a decrease of LVDevP recovery (from $99.8 \pm 7.2 \%$ to $33.3 \pm 6.7 \%$ ) at the end of reperfusion was observed.

\section{Left Ventricular Pressure}

Preconditioning with two short ischemic episodes markedly restored this LVDevP recovery $(93.4 \pm 4.6 \%$ vs $33.3 \pm$ $6.7 \%$ [Figure 4]) while increasing significantly the peak CF 

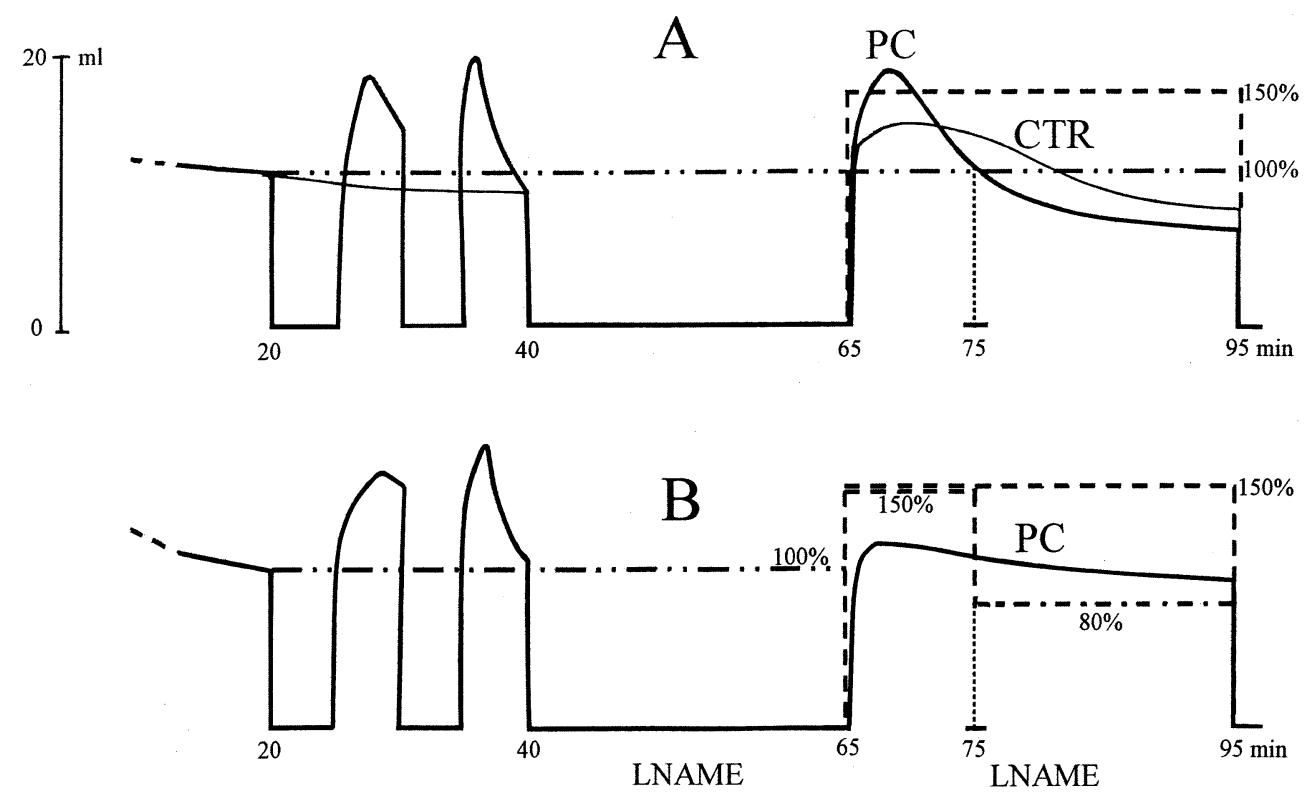

Figure 3. Typical coronary flow traces. A, during the protocols (1) to (4) without drug; B, during the protocols (6) to (8) with L-NAME throughout global low-flow ischemia and reperfusion. Hearts were forcibly reperfused at constant coronary flow (150\% of baseline in groups 4 and 7 ) or reperfused with restricted constant coronary flow $(100 \%$ of baseline in group 3). In group 8 , hearts were forcibly reperfused at $150 \%$ for 10 minutes and reperfused with restricted $\mathrm{CF}(\mathbf{8 0} \%)$ for 20 minutes.

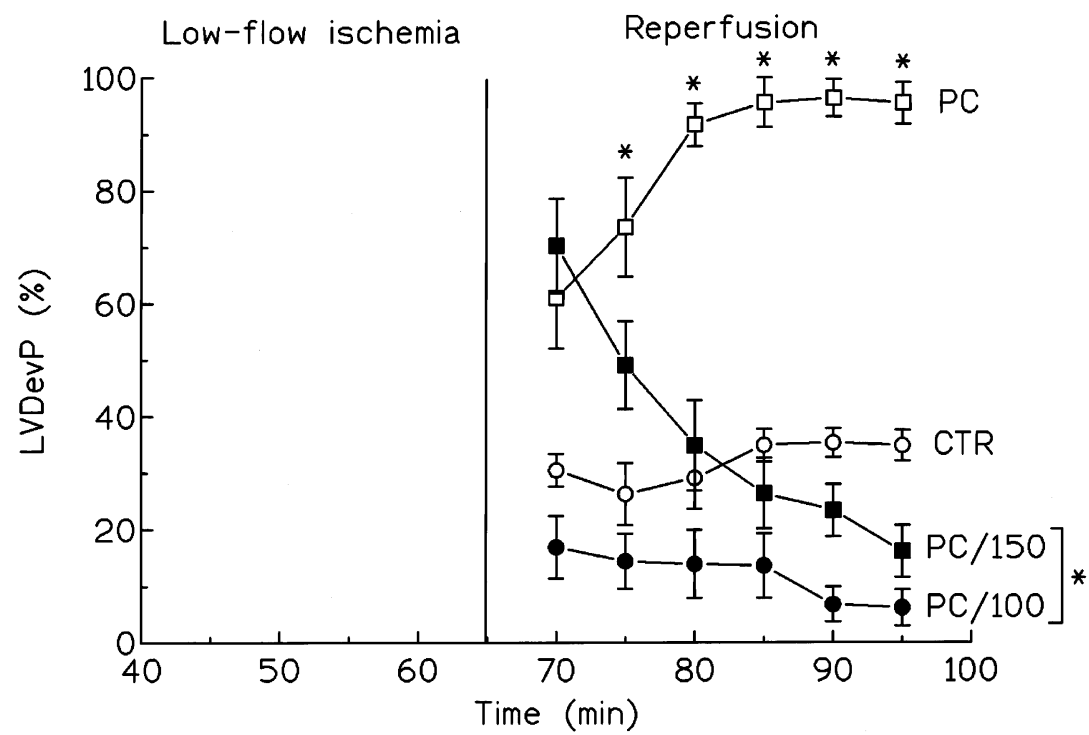

Figure 4. Evolution with time of LVDevP throughout reperfusion following global low-flow ischemia in the control group (CTR), the preconditioned group (PC) with reperfusion at constant pressure, the preconditioned group with restricted constant coronary flow during reperfusion (PC100) and the preconditioned group forcibly reperfused at constant high coronary flow throughout reperfusion (PC 150).

$\left(18.7 \pm 0.8\right.$ vs $\left.14.1 \pm 0.7 \mathrm{~mL} \cdot \mathrm{min}^{-1} \cdot \mathrm{g}^{-1}\right)$ for the same low-flow ischemia duration. Therefore, the coronary RH was increased from 127.2 \pm 8.5 to $165.6 \pm 7.4 \%$ (Figure 3,
A). In the PC/100 group, restricting final peak flow to $100 \%$ of baseline (Figure 3,A) throughout reperfusion suppressed the rise in left ventricular peak developed pressure (Figure 


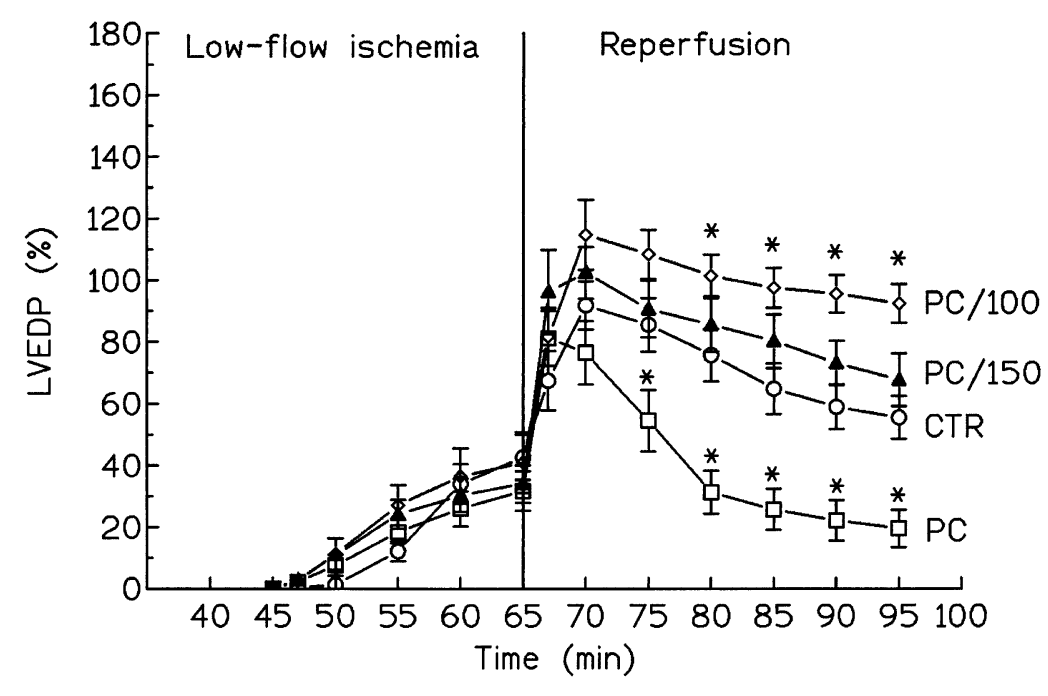

Figure 5. Evolution with time of left ventricular end-diastolic pressure during global low-flow ischemia and reperfusion in the control group (CTR), the preconditioned group reperfused at constant pressure (PC), the preconditioned group reperfused with imposed restricted constant coronary flow (PC 100) and the preconditioned group forcibly reperfused at constant high coronary flow throughout reperfusion (PC 150).

4). In addition, if the hearts are forcibly reperfused to give a peak/baseline flow of $150 \%$ (Figure 3, A) throughout reperfusion, peak developed pressure decreased drastically in the $\mathrm{PC} / 150$ group compared to that in the PC group (Figure 4).

Concomitantly, significant decreases in left ventricular enddiastolic pressure were observed between the PC group and the other three groups during the reperfusion period (Figure 5).

\section{Experiments with L-NAME}

$\mathrm{CF}$ in the PC/L-NAME group returned only to $117.5 \% \pm$ $5.0 \%$ of the preischemic equilibrium level at the beginning of reperfusion (Figure 3, B), compared to $165.6 \% \pm 7.4 \%$ in the PC group without L-NAME. Throughout reperfusion, left ventricular peak developed pressure in the PC/L-NAME group was significantly lower than that in the PC group, but no statistically significant differences were shown between PC/L-NAME, CTR and CTR/L-NAME groups (Figure 6).

In the PC/150/L-NAME group, hearts are forcibly reperfused to give a peak baseline flow of $150 \%$ (Figure 3, B), LVDevP recovered only to $32.8 \% \pm 10.6 \%$ (Figure 7) at the end of reperfusion. In the PC/150 + 80/L-NAME group, hearts are forcibly reperfused to give a peak/baseline flow of $150 \%$ for 10 minutes followed by $80 \%$ for 20 minutes (Figure 3, B) to mimic the evolution of the CF observed in the PC group without L-NAME and at constant pressure (Figure 3,A). Therefore, the rise in left ventricular peak developed pressure was significantly greater throughout reperfusion $(90.3 \% \pm 8.6 \%)$. In addition, the left ventricular end-diastolic pressure in the PC/150 + 80/L-NAME group showed statistically significant differences from the PC/L-
NAME group only at the end of reperfusion but not from the PC/150/L-NAME group (Figure 8).

\section{Ventricular Tachycardia}

In the control hearts, the overall incidence of VT was $2 / 6$ (Table 2). In the majority of hearts, VT developed within the first minute of the reperfusion and the mean duration was $2.5 \pm 2.0$ minutes. The incidence and duration of VT were not significantly affected by ischemic preconditioning. On the contrary, when L-NAME $\left(10^{-5} \mathrm{M}\right)$ was present in the perfusion fluid during low-flow ischemia and reperfusion, the duration of VT was increased in the CTR/L-NAME and PC/L-NAME groups, respectively.

Restricting final peak flow to $100 \%$ of baseline in the $\mathrm{PC} / 100$ group or forcibly reperfusing the hearts to obtain a peak/baseline flow of $150 \%$ in $\mathrm{PC} / 150$ and $\mathrm{PC} / 150 / \mathrm{L}-$ NAME groups give to a rise in incidence and duration of VT so that arrhythmia persisted in $50 \%$ of the hearts at the end of the reperfusion period. However, in the PC/150+ 80/L-NAME group, the duration of VT was significantly reduced and VT are suppressed at the end of reperfusion in all hearts.

\section{Discussion}

Vasodilator mechanisms were classified as endotheliumdependent and endothelium-independent ${ }^{18}$ and endothelium-derived relaxing factor is most likely NO. ${ }^{5}$ Because metabolic and myogenic vasodilation can occur without NO release, they were considered endothelium-independent. ${ }^{19}$ 


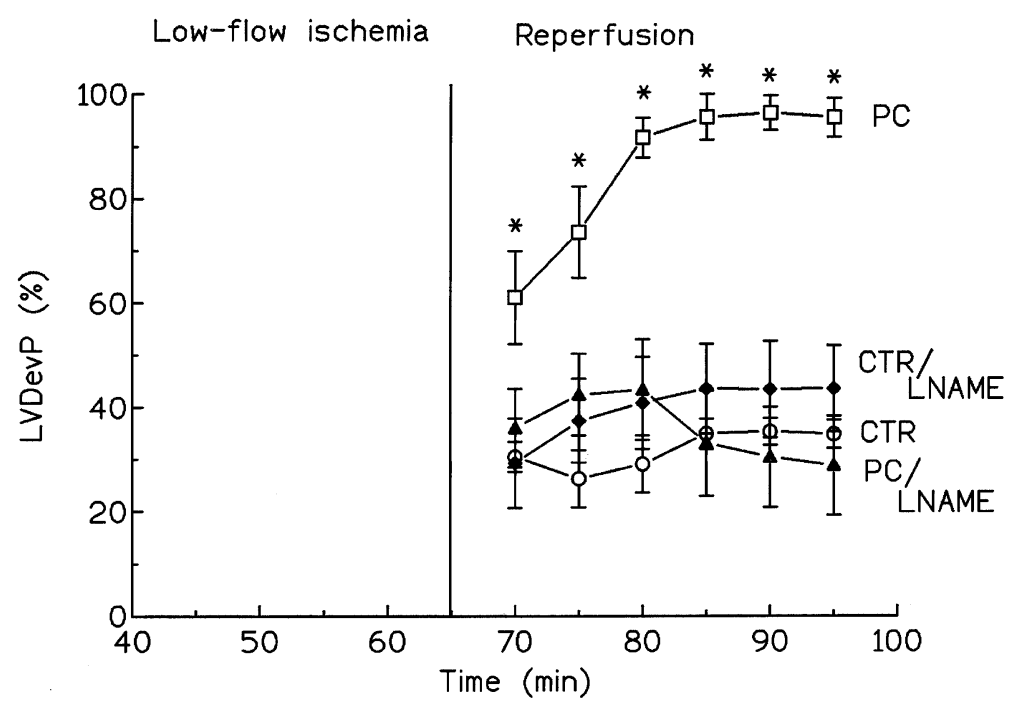

Figure 6. Evolution with time of left ventricular developed pressure, throughout reperfusion in the control group (CTR), the ischemic preconditioned group (PC). Hearts of the CTR/L-NAME and PC/L-NAME groups are perfused with L-NAME during low-flow ischemia and reperfusion. All hearts are reperfused at constant pressure.

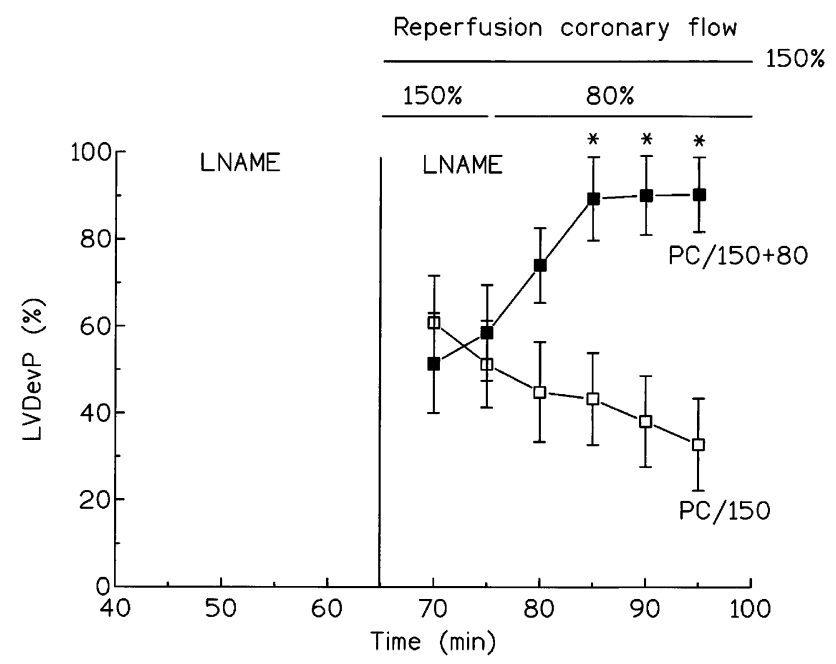

Figure 7. Evolution with time of LVDevP throughout reperfusion in the preconditioned hearts forcibly reperfused at $150 \%$ of baseline coronary flow for 30 minutes (PC/150/L-NAME) and in the preconditioned hearts first forcibly reperfused at $\mathbf{1 5 0} \%$ of baseline CF for 10 minutes and secondly reperfused with a restricted coronary flow (80\% of baseline) for 20 minutes (PC/150 + 80/L-NAME). All hearts were perfused with L-NAME during low-flow ischemia and reperfusion.

The fluid shear stress exerted on the endothelium by the streaming blood represents the major stimulus for a continuous production of NO in vivo. ${ }^{20}$ Myogenic contraction occurs in response to instantaneous increases in transmural pressure, a response that forms the basis of the autoregulatory properties of any given vascular bed. Normally this response is functionally antagonized by the shear stressinduced release of NO. ${ }^{21}$ However, it has been shown that the vasodilation initiated independently of NO release can, in turn, increase the shear stress in the vessels, thus inducing the activation of endothelial NO synthase and prolonging the duration of vasodilation. ${ }^{22}$

The restoration of blood flow to a previously ischemic portion of the myocardium represents an advantage because substrate and oxygen become available to still viable ischemic muscle. ${ }^{23}$ However, there are disadvantages associated with reperfusion, including an enhancement of the speed of necrosis in cells not considered to be viable. Endothelial dysfunction, if present during myocardial reperfusion, will represent an additional problem in the reperfusion injury phenomenon.

The consequences of reperfusion depend on the intensity and duration of the preceding ischemia. In control hearts, we have shown that no contracture is observed when the ischemia time is relatively short (5 minute) while peak reactive hyperemia is high at reperfusion and recovery of LVDevP is maximal. However, prolonged ischemia (25 minute) leads to the development of contracture. Consequently, reactive hyperemia is lesser and the recovery of LVDevP is significantly lower.

Additionally, contracture is increased at reperfusion. Because the compression of the microvasculature has been shown to alter transmural perfusion ${ }^{24}$ this may have contributed to the impairment of CF recovery (low-hyperemia) during reperfusion. However, in all groups ischemic and initial reperfusion contracture amplitudes were not significantly different from CTR. 


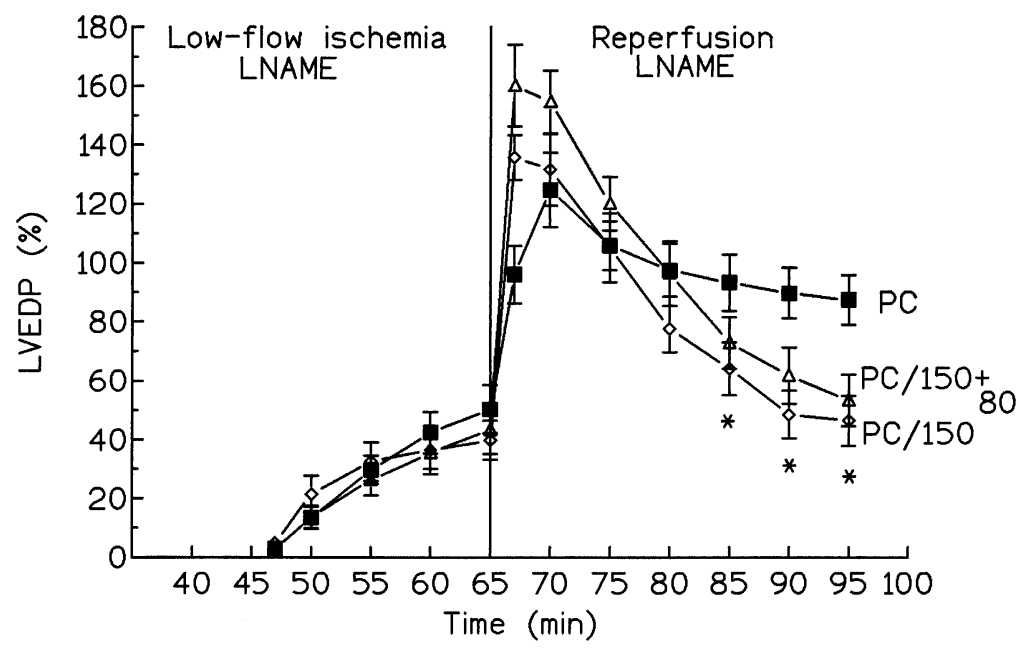

Figure 8. Changes with time of left ventricular end-diastolic pressure in the preconditioned hearts forcibly reperfused at $150 \%$ of baseline CF for 30 minutes (PC/150/L-NAME) and in the preconditioned hearts first forcibly reperfused at $150 \%$ of baseline CF for 10 minutes and secondly reperfused with a restricted $\mathrm{CF}$ ( $80 \%$ of baseline) for 20 minutes (PC/150 + 80/L-NAME), in comparison with preconditioned hearts reperfused at constant pressure (PC/L-NAME). All hearts received L-NAME continuously during low-flow ischemia and reperfusion.

Endothelium-derived NO may be involved in reactive hyperemia following a short duration of coronary no-flow ischemia while it may be less or may not be involved in reactive hyperemia following a prolonged ischemia. ${ }^{25,26}$ However, in a preliminary study, ${ }^{27}$ we found that coronary reactive hyperemia following 25 -minute global low-flow ischemia was suppressed by glibenclamide $\left(\mathrm{I}_{\mathrm{KATP}}\right.$ inhibitor), was decreased with L-NAME (NO pathway inhibitor) or methylene blue (guanylate cyclase inhibitor) while the inhibition of cyclooxygenase with indomethacin failed to affect this coronary reactive hyperemia. These findings suggest that, in our model, the opening of $\mathrm{K}_{\mathrm{ATP}}$ channel contributes to coronary vasodilation in reactive hyperemia and that NO may partly be involved.

In the present study, repeated short preischemic hyperemia increased the postischemic reactive hyperemia and thus improved the postischemic functional recovery in isolated rat hearts. Therefore, we have demonstrated that, although coronary vasodilation in response to shear stress is severely impaired after global low-flow ischemia and reperfusion, it is fully preserved by ischemic preconditioning. This finding indicates that the changes produced in coronary reactive hyperemia and those presumably induced in preconditioning have the same time course, reinforcing the idea that the observed changes in preconditioned hearts are mediated by changes in CF. In contrast, Kitakaze and colleagues $^{28}$ have shown that coronary hyperemia during reperfusion was not different in hearts that did or did not undergo ischemic preconditioning. Moreover, this correlation (hyperemia vs contractile recovery) can be interpreted in a number of ways-cause and effect. Therefore, we
TABLE 2. Ventricular tachycardia (VT): incidence, duration, and end

\begin{tabular}{lcccc}
\hline & & \multicolumn{3}{c}{$\mathbf{V T}$} \\
\cline { 3 - 5 } Groups & $\mathbf{n}$ & Incidence & Duration (min) & End \\
\hline CTR & 6 & $2 / 6$ & $2.5 \pm 2.0$ & $0 / 6$ \\
$\mathrm{PC}$ & 6 & $1 / 6$ & $2.0 \pm 2.0$ & $0 / 6$ \\
$\mathrm{PC} / 100$ & 6 & $5 / 6^{*} \dagger$ & $21.5 \pm 5.5^{*} \dagger$ & $5 / 6^{*} \dagger$ \\
$\mathrm{PC} / 150$ & 6 & $6 / 6^{*} \dagger$ & $20.0 \pm 6.0^{*} \dagger$ & $4 / 6^{*} \dagger$ \\
CTR/L-NAME & 6 & $1 / 6$ & $5.0 \pm 5.0$ & $1 / 6$ \\
$\mathrm{PC} / \mathrm{L}-\mathrm{NAME}$ & 6 & $3 / 6$ & $11.0 \pm 5.0$ & $3 / 6^{*} \dagger$ \\
$\mathrm{PC} / 150 / \mathrm{L}-\mathrm{NAME}$ & 6 & $4 / 6 \dagger$ & $17.0 \pm 6.0^{*} \dagger$ & $3 / 6^{*} \dagger$ \\
$\mathrm{PC} / 150+80 / \mathrm{L}-\mathrm{NAME}$ & 6 & $4 / 6 \dagger$ & $9.0 \pm 4.0$ & $0 / 6$ \\
\hline${ }^{*} P<$ & & & &
\end{tabular}

${ }^{*} P<.05$ vs CTR.

$\mathrm{\dagger} P<.05$ vs $\mathrm{PC}$.

decided to block hyperemia and see whether this blocked preconditioning. In 2 groups, we repeated preconditioning while controlling peak/baseline flow by doing the final reperfusion at constant flow. Thus, in the PC group, restricting final peak flow to $100 \%$ of baseline have reduced left ventricular recovery to the control level, demonstrating as expected that reactive hyperemia is necessary for preconditioning. Moreover, blocking the final reperfusion at constant high CF (150\% of baseline) for 30 minutes reduce drastically left ventricular recovery, suggesting this consistent shear stress may be injurious for endothelial cells.

$\mathrm{NO}$ is emerging as an important cytoprotective agent and may play a pivotal role in myocardial protection both as a trigger and mediator of PC. ${ }^{29}$ The inhibition of NO synthase with L-NAME affects the restoration of reperfusion reactive 
hyperemia afforded by ischemic preconditioning. This finding appears to concur with the involvement claimed by some investigators ${ }^{30}$ but not with the lack of NO contribution reported by others ${ }^{31}$ who have chosen the restoration of contractility or protection from reperfusion arrhythmia as end points.

Nevertheless, hyperemia was suppressed by L-NAME and, concomitantly, LVDevP was significantly reduced in the PC group. Whether this meant that NO-dependent hyperemia but not wall stress was necessary for preconditioning was the key question.

Thus, in the PC/150/L-NAME group, when hearts were forcibly reperfused to give a peak/baseline flow of $150 \%$, they did not recover to better LVDevP as it would have been expected. However, if the time course of hyperemia was restored in the PC/150 + 80/L-NAME group by forcibly reperfusing to $150 \%$ of baseline only for 10 minutes and by restricting final peak flow to $80 \%$ of baseline for 20 minutes, therefore, LVDevP recovered to a high degree despite the presence of L-NAME. An abrupt change in pressure is probably a response to the rapidly attenuated initial peak $\mathrm{CF}$ that could be due to myogenically mediated vasoconstriction $^{32}$ confirming a shear stress endothelial dysfunction relationship.

Finally, the assumption that the changes in the reactive hyperemia are somehow correlated with the degree of cardioprotection give the conclusion that ischemic preconditioning is itself linked with NO-independent vasodilation.

\section{Limitations}

The rat isolated heart perfused with crystalloid and oxygenated buffers has its advantages and limitations like any experimental model. Although the absence of blood cells can limit the comparison of our results to the one obtained in vivo, this model provides a valuable tool to study the local protective mechanisms without any interference with blood-borne elements: the contribution of the platelet-leukocyte aggregates to the pathobiology of ischemia/reperfusion (I/R) injury is therefore excluded. Moreover, studies ${ }^{33}$ have shown that crystalloid cardioplegia does not significantly affect the endothelium-derived nitric oxide-related endothelium-dependent relaxation for up to 4 hours. Therefore, the major cause of injury to endothelium-derived nitric oxide-related function is due to $I / R$ injury to coronary endothelial function ${ }^{34}$ and not due to cardioplegia itself per se. Despite the improved oxygen-carrying capacity of blood cells, the absence of hemoglobin which inactivates luminally released NO is an advantage under the present experimental conditions: findings ${ }^{35}$ provide evidence that the endothelial dysfunction observed in hearts undergoing I/R is due in large part to the reintroduction of oxygen. This reoxygenation causes a burst of superoxide production early in the reperfusion period. Endothelial cells, as well as smooth muscle cells, may serve as the source of these free radicals because neutrophils and monocytes are not present in the model.

\section{References}

1. Olsson RA. Myocardial reactive hyperemia. Circ Res. 1975;37:26370 .

2. Carlsson I, Sollevi A, Wennmalm A. The role of myogenic relaxation, adenosine and prostaglandins in human forearm reactive hyperaemia. J Physiol. 1987;389:147-61.

3. Chilian WM, Layne SM. Coronary microvascular responses to reductions in perfusion pressure: evidence for persistent arteriolar vasomotor tone during coronary hypoperfusion. Circ Res. 1990;66:1227-38.

4. Moncada S, Lorbut R, Bunting S, Vane JR. Prostacyclin is a circulating hormone. Nature. 1978;273:767-8.

5. Palmer RMJ, Ferrige AG, Moncada S. Nitric oxide release accounts for the biological activity of endothelium-derived relaxing factor. Nature. 1987;327:524-6.

6. Yanagisawa M, Kurihara H, Kimura S, Tomobe Y, Kobayashi M, Mitsui Y, et al. A novel potent vasoconstrictor peptide produced by vascular endothelial cells. Nature. 1988;332:411-5.

7. Daut J, Maier-Rudolph W, Von Beckerath N, Mehrke G, Gunther K, Goedel-Meinen L. Hypoxic dilation of coronary arteries is mediated by ATP-sensitive potassium channels. Science. 1990;247:1341-44.

8. Kuo L, Chancellor JD. Adenosine potentiates flow-induced dilation of coronary arterioles by activating atp-sensitive potassium channels in endothelium. Am J Physiol. 1995;269:H541.

9. Jorge PAR, Osaki MR, de Almeida E, Dalva M, Neto LC. Endothelium-dependent coronary flow in ischemia reperfusion. Exp Toxicol Pathol. 1997;49:147-51.

10. Murry CE, Jennings RB, Reimer KA. Preconditioning with ischemia: a delay of lethal cell injury in ischemic myocardium. 1986;74:1124-36.

11. Ovize M, Kloner RA, Hale SL, Przyklenk K. Coronary cyclic flow variations "precondition" ischemic myocardium. Circulation. 1992; 85:779-89.

12. Inoue T, Fujito T, Hoshi K, Sakai Y, Yamaguchi H, Takayanagi K, et al. A mechanism of ischemic preconditioning during percutaneous transluminal coronary angioplasty. Cardiology. 1996;87:216-23.

13. Gianella E, Mochmann H-C, Levi R. Ischemic preconditioning prevents the impairment of hypoxic coronary vasodilatation caused by ischemia/reperfusion. Role of adenosine A1/A3 and bradykinin B2 receptor activation. Circ Res. 1997;81:415-22.

14. Arad M, de Jong JW, de Jonge R, Huizer T, Rabinowitz B. Preconditioning in globally ischemic isolated rat hearts: effect on function and metabolic indices of myocardial damage. J Mol Cell Cardiol. 1996;28:2479-90.

15. Garnier A, Rossi A, Lavanchy N. Importance of the early alterations of energy metabolism in the induction and the disappearance of ischemic preconditioning in the isolated rat heart. J Mol Cell Cardiol. 1996;28: 1671-82.

16. Schjøtt J, Jynge P, Holten T, Brurok H. Ischaemic episodes of less than 5 minutes produce preconditioning but not stunning in the isolated rat heart. Acta Physiol Scand. 1994;150:281-91.

17. Walker MJA, Curtis MJ, Hearse DJ, Campbell RWF, Janse MJ, Yellon DM, et al. The Lambeth Conventions: guidelines for the study of arrhythmia in ischaemia, infarction and reperfusion. Cardiovasc Res. 1988;22:447-55.

18. Parent L, Pare R, Lavallee M. Contribution of nitric oxide to dilatation of resistance coronary vessels in conscious dogs. Am J Physiol. 1992; 262:H10-6.

19. Nishikawa Y, Ogawa S. Importance of nitric oxide in the coronary artery at rest and during pacing in humans. J Am Coll Cardiol. 1997;29:85-92.

20. Lamontagne D, Pohl U, Busse R. Mechanical deformation of the vessel wall and shear stress determine the basal release of endothelium-derived relaxing factor in the intact rabbit coronary vascular bed. Circ Res. 1992;70:123-30.

21. Fleming I, Busse R. Endothelial dysfunction: a novel therapeutic target: NO: the primary EDRF. J Mol Cell Cardiol. 1999;31:5-14. 
22. Gattullo D, Pagliaro P, Marsh NA, Losano G. New insights into nitric oxide and coronary circulation. Life Sci. 1999;65:2167-74.

23. Furchgott RF. Role of endothelium in response of vascular smooth muscle. Cir Res. 1983;53:557-73.

24. Humphrey SM, Thomson RW, Gavin JB. The effect of an isovolumic left ventricular on the coronary vascular competence during reflow after global ischemia in the rat heart. Circ Res. 1981;49:784-91.

25. Kirkeboen KA, Naess PA, Offstad J, Ilebekk A. Effects of regional inhibition of nitric oxide synthesis in intact porcine hearts. Am J Physiol. 1994;266:H1516-27.

26. Tsunoda R, Okumura K, Ishizaka H, Matsunaga T, Tabuchi T, Tayama $S$, et al. Enhancement of myocardial reactive hyperemia with manganese- superoxide dismutase: role of endothelium-derived nitric oxide. Cardiovasc Res. 1996;31:537-45.

27. Barbe C, Rochetaing A, Kreher P. Mechanisms underlying the coronary vasodilation in the isolated perfused hearts of rats submitted to one week of high carbon monoxide exposure in vivo. Inhal Toxicol. 2002;14:101-13.

28. Kitakaze M, Hori M, Takashima S, Sato H, Inoue M, Kamada T. Ischemic preconditioning increases adenosine release and 5'-nucleotidase activity during myocardial ischemia and reperfusion in dogs. Implications for myocardial salvage. Circulation. 1993;87:208-15.
29. Rakhit RD, Edwards RJ, Marber MS. Nitric oxide, nitrates and ischaemic preconditioning. Cardiovasc Res. 1998;43:621-7.

30. Csonka C, Szilvássy Z, Fülöp F, Páli T, Blasig IE, Tosaki A, et al. Classic preconditioning decreases the harmful accumulation of nitric oxide during ischemia and reperfusion in rat hearts. Circulation. 1999; 100:2260-6.

31. Weselcouch EO, Baird AJ, Sleph P, Grover GJ. Inhibition of nitric oxide synthesis does not affect ischemic preconditioning in isolated perfused rat hearts. Am J Physiol. 1995;268:H242-9.

32. Fujita S, Roerig DL, Bosnjak ZJ, Stowe DF. Effects of vasodilators and perfusion pressure on coronary flow and simultaneous release of nitric oxide from guinea-pig isolated hearts. Cardiovasc Res. 1998;38: 655-67.

33. He GW, Yang CQ, Rebeyka IM, Wilson GJ. Effect of neonatal endothelium and smooth muscle to hyperkalemic cardioplegic solution. J Heart Lung Transplant. 1995;14:92-101.

34. Dignan RJ, Dyke CM, Abd-Elfattah AS, Lutz HA, Yeh T, Lee KF, et al. Coronary artery endothelial cell and smooth muscle dysfunction after global myocardial ischemia. Ann Thorac Surg. 1992;53:311-7.

35. Tsao PS, Lefer AM. Time course and mechanism of endothelial dysfunction in isolated ischemic-and hypoxic-perfused rat hearts. Am J Physiol. 1990;259:H1660-6. 